\title{
$p$-SOLVABLE LINEAR GROUPS OF FINITE ORDER
}

\author{
BY \\ DAVID L. WINTER
}

\begin{abstract}
The purpose of this paper is to prove the following result.
THEOREM. Let $p$ be an odd prime and let $G$ be a finite p-solvable group. Assume that $G$ has a faithful representation of degree $n$ over a field of characteristic zero or over $a$ perfect field of characteristic $p$. Let $P$ be a Sylow p-subgroup of $G$ and let $O_{p}(G)$ be the maximal normal p-subgroup of $G$. Then $\left|P: O_{p}(G)\right| \leqq p^{\lambda_{p}(n)}$ where
\end{abstract}

$$
\begin{aligned}
\lambda_{p}(n) & =\sum_{i=0}^{\infty}\left[\frac{n}{(p-1) p^{i}}\right] & \text { if } p \text { is a Fermat prime, } \\
& =\sum_{i=1}^{\infty}\left[\frac{n}{p^{i}}\right] & \text { if } p \text { is not a Fermat prime. }
\end{aligned}
$$

1. Introduction. The above theorem is a generalization of some of the results of J. D. Dixon [1]. Dixon proved the theorem under the stronger hypothesis that $G$ is solvable. Examples are given in [1] showing that the result is best possible for each $n$ and each odd prime $p$. The author thanks Professor Dixon for some helpful suggestions.

2. Preliminaries. $C(S)$ and $C(x)$ will denote the centralizer in the group concerned of, respectively, the set $S$ and the element $x . O_{p}(G)$ and $O_{p^{\prime}}(G)$ denote, respectively, the maximal normal $p$-subgroup of $G$ and the maximal normal subgroup of $G$ whose order is relatively prime to $p . H^{\#}$ is the set of nonidentity elements of the group $H$ while $1_{H}$ is the principal character of $H$. $D$ denotes the field of rational numbers and $\mathbb{C}$ the field of complex numbers.

In $\$ 4$ frequent use is made of the following result of Schur [5].

(2.1) Let $p$ be a prime and let $P$ be a finite p-group which has a faithful representation $X$ of degree $n$ over the complex number field. If the character of $X$ is rationalvalued, then $|P| \leqq p^{f_{p}(n)}$ where

$$
f_{p}(n)=[n /(p-1)]+[n / p(p-1)]+\left[n / p^{2}(p-1)\right]+\cdots .
$$

If $p$ is fixed in the discussion, we shall write $f(n)$ for $f_{p}(n)$.

(2.2) [2, Theorem 2]. Let $\zeta$ be an irreducible complex character of a finite group $N$. Let $A$ be a relatively prime operator group on $N$ such that $A$ fixes $\zeta$. Then:

(a) There exists a unique irreducible character $\eta$ of $A N$ such that $\eta \mid A=\zeta$ and $(\operatorname{det} \eta)(\alpha)=1$ for all $\alpha \in A$.

(b) If $\eta$ satisfies (a), then $\mathscr{D}(\eta)=\mathscr{D}(\zeta)$, and $\eta(\alpha)$ is a rational integer for every $\alpha \in A$.

Received by the editors September 2, 1970.

AMS 1969 subject classifications. Primary 2025, 2075; Secondary 2080.

Key words and phrases. p-solvable linear group, normal p-subgroup.

Copyright (C) 1971, American Mathematical Society 
Definition. The character $\eta$ that satisfies part (a) of (2.2) is called the canonical extension of $\zeta$ to $A N$.

(2.3) Let $\zeta$ be a faithful irreducible complex character of the group $N$ and let $A$ be a cyclic relatively prime operator group on $N$ which fixes $\zeta$. Let $T=C(A) \cap N$ and assume $T \neq N$. Also assume $A$ has odd order and $T=C(\alpha) \cap N$ for all $\alpha \in A^{\#}$. Let $\chi$ be the canonical extension of $\zeta$ to $A N$. Then there exist characters $\lambda, \psi$ of $A T / A$ with $\lambda$ irreducible such that one of the following occurs:

(2.3)(a) $\chi \mid A \times T=\rho \psi+\lambda$.

(2.3)(b) $\chi \mid A \times T=\rho \psi-\lambda$.

Here $\rho$ is the character of the regular representation of $A T / T$.

Proof. By [2, Corollary 5(a)], there exist a sign $\varepsilon= \pm 1$ and an irreducible character $\lambda$ of $T$ such that $\chi(\alpha t)=\varepsilon \lambda(t)$ for all $t \in T$ and all $\alpha \in A^{\#}$ (since $A$ has odd order $\theta_{0}(\alpha)=1$ for all $\alpha \in A$ in the above reference). Let $\lambda$ also denote the character of $A T / A$ whose restriction to $T$ is $\lambda$. Then by the theory of characters of a direct product, $(\chi \mid A T)-\varepsilon \lambda$, as a generalized character of $A \times T$, may be expressed as $\sum_{\theta, \mu} c_{\theta, \mu} \theta \mu$ where $\theta$ and $\mu$ range over the irreducible characters of $A T / T$ and $A T / A$, respectively, and $c_{\theta, \mu}$ is an integer. But since this function vanishes outside $T$, it is easily seen from the inner product formula for $c_{\theta, \mu}$ that $c_{\theta, \mu}$ is independent of $\theta$. Hence $(\chi \mid A T)-\varepsilon \lambda=\rho \psi$ for some generalized character $\psi$ of $A T / A$. Since $\chi \mid A T=$ $\rho \psi+\varepsilon \lambda$ is a character of $A T$, it follows that $\psi$ is 0 or a character of $A T / A$. If $\psi=0$, then by [2, Corollary 5(b)] $T=N$. Hence $\psi \neq 0$ and (2.3) is proved.

3. Initial reductions. We shall describe some of Dixon's results which we may use directly because they do not require the solvability of $G$. By the proofs of Corollaries 1 and 2 of [1], it suffices to prove the theorem assuming the complete reducibility of the given representation. Further, we may assume that the underlying field $\mathfrak{F}$ is algebraically closed. This done, let $G$ be a counterexample to the theorem of minimal order. Let $P$ denote a fixed Sylow $p$-subgroup of $G$ with, say, $|P|=p^{a}$. Let $n$ be the smallest positive integer such that $G$ has a faithful completely reducible representation of degree $n$ over $\mathfrak{F}$ and $\left|P: O_{p}(G)\right|>p^{\lambda_{p}(n)}$. Let $X$ denote such a representation and let $\chi$ be its character. As in [1, pp. 547-548] it may be shown that

(3.1) $X$ is irreducible and primitive.

If $\mathfrak{F}$ has characteristic $p, X$ may be lifted to a representation over $\mathfrak{E}[6$, Theorem 6]. Hence we may assume that $\mathfrak{F}=\mathfrak{C}$ and shall do so from now on.

(3.2) $G$ has a series of normal subgroups $O_{p}(G)<N_{1}<G$ where $N_{1} / O_{p}(G)$ $=O_{p^{\prime}}\left(G / O_{p}(G)\right)$ and $G / N_{1}$ is a p-group.

Proof. Let $P_{1} / N_{1}=O_{p}\left(G / N_{1}\right)$ and suppose $P_{1} \neq G$. Then $P N_{1} \neq G$ and by induction $O_{p}(G)<O_{p}\left(P N_{1}\right)$. Since $P_{1} \leqq P N_{1}, P_{1}$ normalizes $O_{p}\left(P N_{1}\right) \cap P_{1} \geqq O_{p}(G)$. Since $P_{1} \triangleleft G, O_{p}\left(P_{1}\right) \triangleleft G$ and this implies $O_{p}(G)=O_{p}\left(P N_{1}\right) \cap P_{1}$. Hence $O_{p}\left(P N_{1}\right) P_{1} / N_{1}$ $=\left(O_{p}\left(P N_{1}\right) N_{1} / N_{1}\right) \times\left(P_{1} / N_{1}\right)$ which is contradictory to Lemma 1.2.3 of HallHigman [3]. Hence $P_{1}=G$ as desired. 
(3.3) $\left|P: O_{p}(G)\right|=p^{\lambda_{p}(n)+1}$.

Proof. $G$ contains a normal subgroup $H$ of index $p$ containing $N_{1}$. Since $O_{p}(H) \triangleleft G, \quad O_{p}(H) \leqq O_{p}(G)$. By minimality of $|G|, \quad p^{\lambda_{p}(n)} \geqq\left|P \cap H: O_{p}(H)\right|$ $\geqq\left|P: O_{p}(G)\right| \mid p>p^{\lambda_{p}(n)-1}$, which proves (3.3).

(3.4) $N_{1}=O_{p}(G) \times N$ where $N$ is a normal p-complement of $G$.

Proof. By [4, Lemma 1] it suffices to show that $O_{p}(G)$ is contained in the Frattini subgroup of $G$. Let $M$ be a maximal subgroup of $G$ not containing $O_{p}(G)$. Then $G=O_{p}(G) M$. Now $M$ and $O_{p}(G)$ normalize $O_{p}(G) O_{p}(M)$. Hence $O_{p}(M)$ $=O_{p}(G) \cap M$. Let $|M|=p^{s} g^{\prime}$ and $|G|=p^{a} g^{\prime}, p \nmid g^{\prime}$. By minimality of $|G|$, $|G|=\left|O_{p}(G)\right||M| /\left|O_{p}(G) \cap M\right| \leqq\left|O_{p}(G)\right| p^{\lambda_{p}(n)} g^{\prime}$. This contradicts (3.3) and (3.4) is proved.

(3.5) $G=P N, P \cap N=\langle 1\rangle, \chi \mid N$ is irreducible, $O_{p}(G)=\langle 1\rangle$ and $\lambda_{p}(n)=a-1$.

Proof. Suppose that $\chi \mid N$ is reducible. Since $G / N$ is a $p$-group, there is a sequence of normal subgroups of $G, G=P_{0}>P_{1}>\cdots>P_{k}=N$, such that $\left|P_{i}: P_{i+1}\right|=p$. Choose $i$ so that $\chi \mid P_{i}$ is irreducible and $\chi \mid P_{i+1}$ is reducible. Then it is well known that $\chi \mid P_{i+1}$ is a sum of $p$ distinct conjugate characters. This contradicts the primitivity of $X$ and so $\chi \mid N$ is irreducible. Therefore $p \nmid \chi(1)$ and since $O_{p}(G) \leqq C(N)$, $O_{p}(G) \leqq Z(G)$ by Schur's lemma. Hence $\chi \mid O_{p}(G)=\chi(1) \lambda$ where $\lambda$ is a linear character of $O_{p}(G)$. Since $p \nmid \chi(1), \chi \mid P$ contains a linear character $\mu$ of $P$ and $\mu \mid O_{p}(G)=\lambda$. Regarding $\mu$ as a character of $G / N$, we see that $\bar{\mu} \chi$ is a faithful character of $G / O_{p}(G)$ of degree $n$. If $O_{p}(G) \neq\langle 1\rangle$, minimality of $|G|$ yields a contradiction. Hence $O_{p}(G)$ $=\langle 1\rangle$ and the last statement of (3.5) follows from (3.3).

4. Completion of the proof. The canonical extension of $\chi \mid N$ to $G$ must be faithful since its kernel is a $p$-group and $O_{p}(G)=\langle 1\rangle$. Hence we may assume that $\chi$ is the canonical extension of $\chi \mid N$ to $G$ and shall do so from now on. By (2.2)(b) $\chi$ is rational-valued on $P$ and so by (2.1), we may assume $p$ is not a Fermat prime. In particular, $p \geqq 7$ and it is easily seen that $f(t)=\lambda_{p}((p /(p-1)) t)$ for any positive rational number $t$.

We now let $w$ be an element of $Z(P)$ of order $p$ such that if $v$ is any element of $Z(P)$ of order $p\left(\chi \mid\langle v\rangle, 1_{\langle v\rangle}\right)_{\langle v\rangle} \leqq\left(\alpha\left|\langle w\rangle, 1_{\langle w\rangle}\right\rangle_{\langle w\rangle}\right.$. Let $A=\langle w\rangle$ and let $T=C(w) \cap N$. Then $P$ normalizes $T$.

Let $1=\theta_{1}, \theta_{2}, \ldots, \theta_{p}$ be the distinct linear characters of $A \times T$ whose kernels contain $T$. Since $w \in Z(P T)$, we may write $\chi \mid P T=\alpha_{1}+\cdots+\alpha_{p}$ where $\alpha_{i}$ is a character of $P T$ (or $\alpha_{i}=0$ is possible) such that $\alpha_{i} \mid A$ is a multiple of $\theta_{i} \mid A$ for $i=1, \ldots, p$. By (2.2)(b), $\chi$ is invariant under the Galois group $\mathbb{B}=\operatorname{Gal}\left(\mathfrak{D}(\varepsilon) / \mathfrak{D}\left(\varepsilon^{p a}\right)\right)$ where $\varepsilon$ is a primitive $|G|$ th root of unity. It follows that $\alpha_{2}, \ldots, \alpha_{p}$ is a full set of conjugates of $\alpha_{2}$ under this group and $\alpha_{2}+\cdots+\alpha_{p}$ as well as $\alpha_{1}$ are rational valued on $P$. The remainder of the proof is split into the two possible cases $\chi \mid A \times T=\rho \psi+\lambda$ or $\chi \mid A \times T=\rho \psi-\lambda$ as described in (2.3).

Assuming that the first case holds, we have $\alpha_{1} \mid T=\psi+\lambda$ and $\alpha_{i} \mid T=\psi$ for $i=2, \ldots, p$. Let $\cup=\operatorname{ker}\left(\alpha_{2}+\cdots+\alpha_{p}\right) \cap P$. We require the 
LEMMA. $(\psi, \lambda)_{T} \neq 0$. In particular, $\psi(1) \geqq \lambda(1)$.

Suppose $(\psi, \lambda)_{T}=0$. Then $(\chi, \lambda)_{T}=1 . \lambda \mid T$ is fixed by $P$ because $P$ fixes $\chi \mid T$ and $\lambda$ is the only irreducible constituent of $\chi \mid T$ whose multiplicity is not a multiple of $p$. It follows from Clifford's theorem that $\chi \mid P T$ contains a unique irreducible constituent $\beta$ which is an extension of $\lambda \mid T$. Because $\chi \mid P T$ is invariant under (S) so is $\beta$ and $\beta$ is therefore rational on $P$.

Let $S$ be such that $\operatorname{ker} \beta \cap P \leqq S \leqq P$ and $S \operatorname{ker} \beta / \operatorname{ker} \beta=O_{p}(P T / \operatorname{ker} \beta)$. Then $S T / \operatorname{ker} \beta=S \operatorname{ker} \beta / \operatorname{ker} \beta \times T \operatorname{ker} \beta / \operatorname{ker} \beta$. Since $\beta \mid T$ is irreducible, $\beta \mid S=\beta(1) \mu$ for some linear character $\mu$ of $S$. Since $\beta$ is rational on $S$, we must have $S \leqq \operatorname{ker} \beta$ and therefore $S=\operatorname{ker} \beta \cap P$. By our induction hypothesis, $|P: S| \leqq p^{\lambda_{p}(\beta(1))}$. Since $\lambda(1)$ $=\beta(1)$, we have shown $|S| \geqq p^{a-\lambda_{p}(\lambda(1))}$.

On the other hand, by Schur's theorem (2.1), $|P: \cup| \leqq p^{f((p-1) \psi(1))}=p^{\lambda_{p}(p \psi(1))}$ $=p^{\lambda_{p}(n-\lambda(1))}$. Therefore,

$$
\begin{aligned}
p^{a} & \geqq|S \cup|=\frac{|S||\cup|}{|S \cap \cup|} \geqq \frac{p^{a-\lambda_{p}(\lambda(1))} p^{a-\lambda_{p}(n-\lambda(1))}}{|S \cap \cup|} \\
& \geqq \frac{p^{2 a-\lambda_{p}(\lambda(1)+n-\lambda(1))}}{|S \cap \cup|}=\frac{p^{a+1}}{|S \cap \cup|}
\end{aligned}
$$

by (3.5). This shows that $|S \cap \cup| \neq 1$. Let $u$ be an element of $S \cap \cup \cap Z(P)$ of order $p$. By our choice of $w, \lambda(1)+\psi(1) \geqq\left(\chi, 1_{\langle u\rangle}\right)_{\langle u\rangle} \geqq \lambda(1)+(p-1) \psi(1)$. This contradiction proves the lemma.

Now suppose $U \neq\langle 1\rangle$. Let $u \in \cup \cap Z(P)$ have order $p$. Then $\psi(1)+\lambda(1)$ $\geqq\left(\chi, 1_{\langle u\rangle}\right)_{\langle u\rangle} \geqq(p-1) \psi(1) \geqq(p-2) \psi(1)+\lambda(1)$ by the lemma. This is a contradiction and so $\cup=\langle 1\rangle$. As in the proof of the lemma, $|P|=|P: \cup| \leqq p^{\lambda_{p}(n-\lambda(1))} \leqq p^{\lambda_{p}(n)}=p^{a-1}$. This is a contradiction and the proof in the first case is complete.

Assume now that (2.3)(b) holds for $\chi$ and $A$, i.e., $\chi \mid A \times T=\rho \psi-\lambda$. Write $\rho=1+\theta$ where $\theta$ is the sum of the nonprincipal linear characters of $A T / T$. Because $\chi \mid A T$ is a character of $A T$, it must be a linear combination of irreducible characters of $A T$ with positive integral coefficients and so $\lambda$ must be a constituent of $\rho \psi$. Let $r=(\rho \psi, \lambda)_{A T} \geqq 1$. Then $\psi=r \lambda+\psi_{1}$ where $\psi_{1}=0$ or $\psi_{1}$ is a character of $A T / A$ which does not contain $\lambda$. Therefore $\chi \mid A T=(1+\theta)\left(r \lambda+\psi_{1}\right)-\lambda=(r-1) \lambda+r \theta \lambda+\rho \psi_{1}$. It follows that $\alpha_{1} \mid A T=(r-1) \lambda+\psi_{1}$ and $\alpha_{i} \mid A T=r \theta_{i} \lambda+\theta_{i} \psi_{1}$ for $i=2, \ldots, p$.

Assume first that $\psi_{1} \neq 0$. Because $\lambda \mid T$ is the only irreducible constituent of $\chi \mid T$ of multiplicity congruent to $-1 \bmod p, \lambda \mid T$ is invariant under $P$. Therefore by Clifford's theorem, we may write, for $i>1, \alpha_{i}=\mu_{i}+\nu_{i}$ where $\mu_{i}$ and $\nu_{i}$ are characters of $P T$ such that $\mu_{i} \mid T=r \lambda$ and $\nu_{i} \mid T=\psi_{1}$. Since $T$ is a $p^{\prime}$-group, $\lambda \mid T$ is invariant under (S). It follows that $\mu_{2}, \ldots, \mu_{p}$ is a full set of conjugates under $\mathscr{S}$ and that $\mu=\mu_{2}+\cdots+\mu_{p}$ is rational valued on $P$. Since $\chi, \alpha_{1}$ and $\mu$ are rational on $P$, $\chi-\alpha_{1}-\mu=\nu_{2}+\cdots+\nu_{p}=\nu$ is rational on $P$. Let $U=\operatorname{ker} \mu \cap P, V=\operatorname{ker} \nu \cap P$ and $S=\operatorname{ker} \alpha_{1} \cap P$. By (2.1), $|\cup| \geqq p^{a-f((p-1) r \lambda(1))}, \quad|V| \geqq p^{a-f\left((p-1) \psi_{1}(1)\right)}$ and $|S|$ $\geqq p^{a-f\left(\alpha_{1}(1)\right)}$. By our choice of $w$, we must have $S \cap \cup=\langle 1\rangle$ and $S \cap V=\langle 1\rangle$. This leads to two inequalities. 
First,

$$
1=|S \cap \cup|=|S||\cup| /|S \cup| \geqq p^{2 a-\left\{f\left(\alpha_{1}(1)\right)+f((p-1) r \lambda(1))\right\}} / p^{a} .
$$

Hence,

$$
\begin{aligned}
0 & \geqq a-\left\{f\left(\alpha_{1}(1)\right)+f((p-1) r \lambda(1))\right\} \geqq a-f\left(\alpha_{1}(1)+(p-1) r \lambda(1)\right) \\
& =a-\lambda_{p}\left((p /(p-1)) \alpha_{1}(1)+p r \lambda(1)\right) .
\end{aligned}
$$

Since $\lambda_{p}(n)=a-1$ by (3.5), this implies $(p /(p-1)) \alpha_{1}(1)+p r \lambda(1)>n$. Because $\alpha_{1}(1)$ $=(r-1) \lambda(1)+\psi_{1}(1)$ and $n=(p r-1) \lambda(1)+p \psi_{1}(1)$, this inequality reduces to

$$
(p r-1) \lambda(1)>p(p-2) \psi_{1}(1) \text {. }
$$

Second,

$$
1=|S \cap V|=|S||V| /|S V| \geqq p^{a-f\left(\alpha_{1}(1)\right)} p^{a-f\left((p-1) \psi_{1}(1)\right)} / p^{a} .
$$

Hence

$$
\begin{aligned}
0 & \geqq a-\left\{f\left(\alpha_{1}(1)\right)+f\left((p-1) \psi_{1}(1)\right)\right\} \geqq a-f\left(\alpha_{1}(1)+(p-1) \psi_{1}(1)\right) \\
& =a-\lambda_{p}\left((p /(p-1)) \alpha_{1}(1)+p \psi_{\perp}(1)\right) .
\end{aligned}
$$

This implies $(p /(p-1)) \alpha_{1}(1)+p \psi_{1}(1)>n$ which reduces to

$$
p \psi_{1}(1)>\left(p^{2} r-2 p r+1\right) \lambda(1) .
$$

Combining (1) and (2), we have $\left(p^{2} r-2 p r+1\right) \lambda(1)(p-2)<p(p-2) \psi_{1}(1)$ $<(p r-1) \lambda(1)$ and hence $\left(p^{2} r-2 p r+1\right)(p-2)<p r-1$. This is equivalent to $\left(p^{3} r-4 p^{2} r\right)+(p+3 p r-1)<0$ which is impossible both terms on the left being positive.

Finally, take $\psi_{1}=0$. Then $\alpha_{1}\left|A \times T=(r-1) \lambda, \alpha_{i}\right| A \times T=r \theta_{i} \lambda$ for $i>1$ and $\chi \mid T$ $=(p r-1) \lambda$. In particular, $\lambda$ is a faithful character of $T$. Therefore $\operatorname{ker} \alpha_{1}$ is a $p$ group and setting $R=O_{p}(P T)$, we have $|P: R| \leqq p^{\lambda_{p}\left(\alpha_{1}(1)\right)}$ or $|R| \geqq p^{a-\lambda_{p}\left(\alpha_{1}(1)\right)}$.

We have $\left(\chi \mid A, 1_{A}\right)_{A}=\alpha_{1}(1)<\alpha_{2}(1)$. By our choice of $w$, ker $\alpha_{2} \cap P=\langle 1\rangle$. It follows that $\alpha_{2}+\cdots+\alpha_{p}$ is the character of a faithful matrix representation $Y$ of $P T$ over $\mathbb{E}$ with $\alpha_{2}+\cdots+\alpha_{p}$ rational on $P$. By a suitable choice of basis of the underlying vector space we may assume that $Y(t)=A(t) \otimes I_{(p-1) r}$. Here $A(t)$ is an irreducible matrix representation of $T$ with character $\lambda$ and $I_{s}$ denotes the $s \times s$ identity matrix. Since $R$ centralizes $T$, it is easily verified using Schur's lemma that $Y(r)=I_{\lambda(1)} \otimes B(r), r \in R$, where $B(r)$ is a faithful representation of $R$ with character $\left(\alpha_{2}+\cdots+\alpha_{p}\right) / \lambda(1)$. By (2.1), $|R| \leqq p^{f((p-1) r)}=p^{\lambda_{p}(p r)}$. Combining this with our previous inequality, we get $a \leqq \lambda_{p}\left(\alpha_{1}(1)\right)+\lambda_{p}(p r) \leqq \lambda_{p}((r-1) \lambda(1)+p r)$. By (3.5), $(r-1) \lambda(1)+p r>n=(r-1) \lambda(1)+(p-1) r \lambda(1)$ or $p r>(p-1) r \lambda(1)$. This implies $\lambda(1)$ $<p /(p-1)<2$ and so $\lambda(1)=1$. But then $\chi \mid T=\chi(1) \lambda$ and $T=Z(G)$. Since $w$ acts fixed-point-free on $N / T$, a well-known result of Thompson yields that $N / T$ is nilpotent. $G$ is therefore solvable and the result of [1] completes the proof. 


\section{REFERENCES}

1. J. D. Dixon, Normal p-subgroups of solvable linear groups, J. Austral. Math. Soc. 7 (1967), 545-551. MR 37 \#6373.

2. G. Glauberman, Correspondences of characters for relatively prime operator groups, Canad. J. Math. 20 (1968), 1465-1488. MR 38 \#1189.

3. P. Hall and G. Higman, The p-length of p-soluble groups and reduction theorems for Burnside's problem, Proc. London Math. Soc. (3) 6 (1956), 1-42. MR 17, 344.

4. L. G. Kovács, On finite soluble groups, Math. Z. 103 (1968), 37-39. MR 36 \#6506.

5. I. Schur, Über eine Klasse von endlichen Gruppen linearer Substitutionen, S.-B. Preuss. Akad. Wiss: 1905, 77-91.

6. R. G. Swan, The Grothendieck ring of a finite group, Topology 2 (1963), 85-110. MR 27 \#3683.

Michigan State University,

East Lansing, Michigan 48823 